\title{
267 DUAL CHECKPOINT BLOCKADE OF CD47 AND PD-L1 USING AN AFFINITY-TUNED BISPECIFIC ANTIBODY MAXIMIZES ANTI-TUMOR IMMUNITY AND IMPROVES THERAPEUTIC WINDOW
}

Shih-Hsun Chen, Pawel Dominik, Jessica Stanfield, Sheng Ding, Wenjing Yang, Nadia Kurd*, Ryan Llewellyn, Jonathan Heyen, Carole Wang, Zea Melton, Kevin Lindquist, Thomas Van Blarcom, Javier Chaparro-riggers, Shahram Salek-Ardakani. Pfizer, San Diego, CA, USA

Background $\mathrm{T}$ cell checkpoint immunotherapies have shown promising results in the clinic, but most patients remain nonresponsive. CD47-SIRP $\alpha$ myeloid checkpoint blockade has shown early clinical activity in hematologic malignancies. However, CD47 expression on peripheral blood limits $\alpha$ CD47 antibody selectivity and thus efficacy in solid tumors.

Methods To improve the antibody selectivity and therapeutic window, we developed a novel affinity-tuned bispecific antibody targeting CD47 and PD-L1 to antagonize both innate and adaptive immune checkpoint pathways. This PD-L1-targeted CD47 bispecific antibody was designed with potent affinity for PD-L1 and moderate affinity for CD47 to achieve preferential binding on tumor and myeloid cells expressing PD-L1 in the tumor microenvironment (TME).

Results The antibody design reduced binding on red blood cells and enhanced selectivity to the TME, improving the therapeutic window compared to $\alpha \mathrm{CD} 47$ and its combination with $\alpha$ PD-L1 in syngeneic tumor models. Mechanistically, both myeloid and $\mathrm{T}$ cells were activated and contributed to antitumor activity of $\alpha$ CD47/PD-L1 bispecific antibody. Distinct from $\alpha \mathrm{CD} 47$ and $\alpha \mathrm{PD}-\mathrm{L} 1$ mono- or combination therapies, single-cell RNA sequencing (scRNA-seq) and gene expression analysis revealed that the bispecific treatment resulted in unique innate activation, including Pattern Recognition Receptor (PRR)-mediated induction of type I IFN pathways and antigen presentation in DCs and macrophage populations. Furthermore, treatment increased the Tcf7 + stem-like CD8 T cell population in the TME and promoted its differentiation to an effector-like state. Consistent with mouse data, the compounds were well tolerated and demonstrated robust myeloid and $\mathrm{T}$ cell activation in non-human primates (NHPs). Notably, RNAseq analysis in NHPs provided evidence that the innate immune activation was mainly contributed by CD47-SIRP $\alpha$ but not PD-L1-PD-1 blockade from the bispecific antibody.

Conclusions These findings provide novel mechanistic insights into how myeloid and $\mathrm{T}$ cells can be uniquely modulated by the dual innate and adaptive checkpoint antibody and demonstrate its potential in clinical development (NCT04881045) to improve patient outcomes over current PD-(L)1 and CD47-targeted therapies.

http://dx.doi.org/10.1136/jitc-2021-SITC2021.267 